\title{
Green Technologies for Management on Railroad Transportation
}

\author{
Dmitry Efanov ${ }^{1,2,4^{*}}$, German Osadchiy ${ }^{2}$, Dmitry Plotnikov ${ }^{1}$, Aushra Banite $^{1}$, Begmat Norov $^{3}$ \\ and Davron Ruziev ${ }^{4}$ \\ ${ }^{1}$ Peter the Great St. Petersburg Polytechnic University, 29 Plotytechnicheskaya str., St. Petersburg, \\ Russian Federation \\ ${ }^{2}$ Scientific and Technical Center "Integrated Monitoring Systems" LLC, 4-K Fuchika str., \\ St. Petersburg, Russian Federation \\ ${ }^{3}$ Tashkent Institute of Irrigation and Agricultural Mechanization Engineers, 39 str. Kari Niyaziy, \\ Tashkent, Uzbekistan, \\ ${ }^{4}$ Russian University of Transport (MIIT), 9-9 Obraztsova str., Moscow, Russian Federation
}

\begin{abstract}
Technique and technology development allows us to discuss the possibility of crucial changes into a conservative way of railroad transportation management. One of the options regarding the train control system is aimed at denial of the classic approach being connected with the implementation of a traditional automation kit. The authors of this article are being presented with the conception of a green IT railroad rolling stock control system called Green Interlocking. The above approach helps us rely on effective inexhaustible energy sources and modern know-how of data processing plus important technologies management.
\end{abstract}

\section{Introduction}

As is known, technique with technology evolution movement is being conducted via spiral way all over. Some of the scientific solutions that were presented tens or sometimes hundreds of years back are being gradually expelled by modern, more effective ones either those ideas have new life based on current IT tech boost. Consequently, each ongoing spire of technological advance should help us not just to accelerate some of the scientific solutions and approaches only, but, really, to expand the field of choice reckoning methods of task realization plus, as a result, we may obtain the more cost-effective and "clever" outcome. For example, the idea of new transistors development, wide implemented within CMOS devices of nowadays automation and calculation technique was patented by Julius Edgar Lilienfeld back in 1926-1928. Meanwhile, the first-ever field-effect transistor (FET) was presented thirty years later, in 1960, with the advancement of the isolated shutter was prepared by Carver Mead. In 1977 George Makkalahem from Bell Laboratories showed that FET application might drastically improve the execution speed of computation technique. New bipolar transistors hit the market plus logical elements with microchips having a relatively low grade of integration during the time of fast-growing demand for

\footnotetext{
${ }^{*}$ Corresponding author: tres-4b@yandex.ru
} 
field-effect transistors. Contemporary developed potential in the sphere of discrete systems with a high and ultrahigh grade of integration and possibility to imply those gadgets with rekey boarding logic is widespread in design and arrangement of automated management, including railroad transportation branch [1]. Scope of works aimed at energy wastage reduction, per various components, are being constantly conducted together with green IT serious contribution [2].

Spiral technique development is the matter within management systems of critical technological performance of railroad transportation (Figure 1). For instance, in XIX Century, the idea of centralized management within railroad stations was completed via ridged with flexible control leverage of operator, and hundred years later, it was transformed into relay devices [3]. As for today, centralized management with electrical power supply provision is being performed all over the systems of railroad automation, including microelectronics with microprocessors hardware components [4, 5]. During the transforming period from the hand gear mechanisms toward automated electrical schemas of transportation control, some decentralized options of equipment placement based on energy power sources locations were being appeared. First-ever automated systems of railroad management presentation belong to the initial part of XX Century. Technology with that period technique had low effectiveness of such an option implementation reckoning train management system. Hence, hundred years later, within the Era of IT Solutions plus alternative sources of energy options, the inkling of the decentralized location of equipment installation received new boost acceleration [6].

Green IT implementation is widespread in the present-day world in such spheres as construction and information with technical tasks [2, 7, 17-20]. The above conception appeared back in 1992 and was attached to an ecological targeted computerized technique branch. Those essential trends of that green IT option were as follows: reduction of hazardous material use, maximum effectiveness enhance, energy consumption cutting down, service period of sites prolongation, maintenance prospect improvement, and, for sure, the recycling possibility. In this case, alternative energy with green IT such as wind power, hydro energy, tides with solar power, and geothermal sources with bio fuel enhancements are very important. Based on the IT specialist report, we shall have an alternative energy option privilege worldwide by the end of 2021 [8].

\section{Methods}

The development of the railroad rolling stock control system «Green Interlocking» is based on the analysis of the following paradigms: 'make-depends centralization,' 'energy power supply centralization,' and 'cable network for data transmission application.' In this particular option, energy consumption optimization, together with green IT, is being left out of the equation. Realization of smart, cost-effective option regarding train management kit is possible in the event of those three paradigms concentration performance, i.e. 'data processing directly at sites,' 'self-contained power,' plus 'wireless network' being proposed by authors [6]. Following the aforesaid, we may fulfil this required 'green interlocking' conception. A crucial advantage of this approach is evident via low energy utilization, cost cutouts plus balanced resources consumption with ecological technologies implementation as well as time and some extra equipment elimination (rejection of rather costly physical servers with trackside assets such as impedance bonds installation). 


\section{Results and Discussion}

From every nowadays realization of energy-effective systems, there is only one about to be reality reckoning railroad transportation function. We speak about wind plus solar energy treatment. For example, from the beginning of 2017, the entire trains of Netherlands were being transformed into wind energy consumption [9]. In Figure 2, we may see the conceptual schema of the 'Green Interlocking' sample. This particular schema is based on up-to-date technologies applications such as fog computing with big data, including IoT plus blockchain and neural network [10 - 14].

The system architecture consists of several hierarchy levels, including a grassroots level of interfacing with trackside process equipment and the one of wireless communication with the operational control sites as well as the operational control stage itself formed via functional control modules (FCMs) and a wireless data network based on the abovementioned. The function modules are to be connected directly to control units and contain a management and built-in monitoring microcontroller and a signal transmitter. Thus, the functional modules are distributed over stations with railroad hauls and form a distributed computer network based on fog computing tech. The entire relationships are being checked right here at the lower execution stage. The access to the sensors is being arranged via a cyber-protected wireless channel using the developed cryptographic protocol following the genetic algorithm in the compiler to ensure maximum energy efficiency [15], from a special automated workstation (AWS) by a traffic operator at a site (analogue of a station master-on-duty). In the long run, the functions of an operator are to be gradually eliminated and should be used by an automation dispatcher for the organization of rational train movement control modes.

There can be a phased transition to reducing the number of trackside process equipment of railway automation while introducing the Green Interlocking concept. At the initial stage, conventional means are being preserved in the form of track circuits, traffic lights, railroad switches, impedance bonds, etc. At the second step, colour light signaling is being abandoned via transferring the required velocity to on-board locomotive units. At the third stage, track circuits are being eliminated. The number of impedance bonds is being minimized, mobile units are being positioned using the radio channel, and the integrity of the rail track is being monitored based on smart fiber-optic systems. Thus, the final stage is only connected with the preservation of railroad points automatic control devices (it only makes sense in case of changing engine types being applied plus the ratings of their operation currents and voltages) and formation of own protected IoT for train dispatching service. The transition from AC intended for control with subsequent rectification to DC capability is gradually completed. 


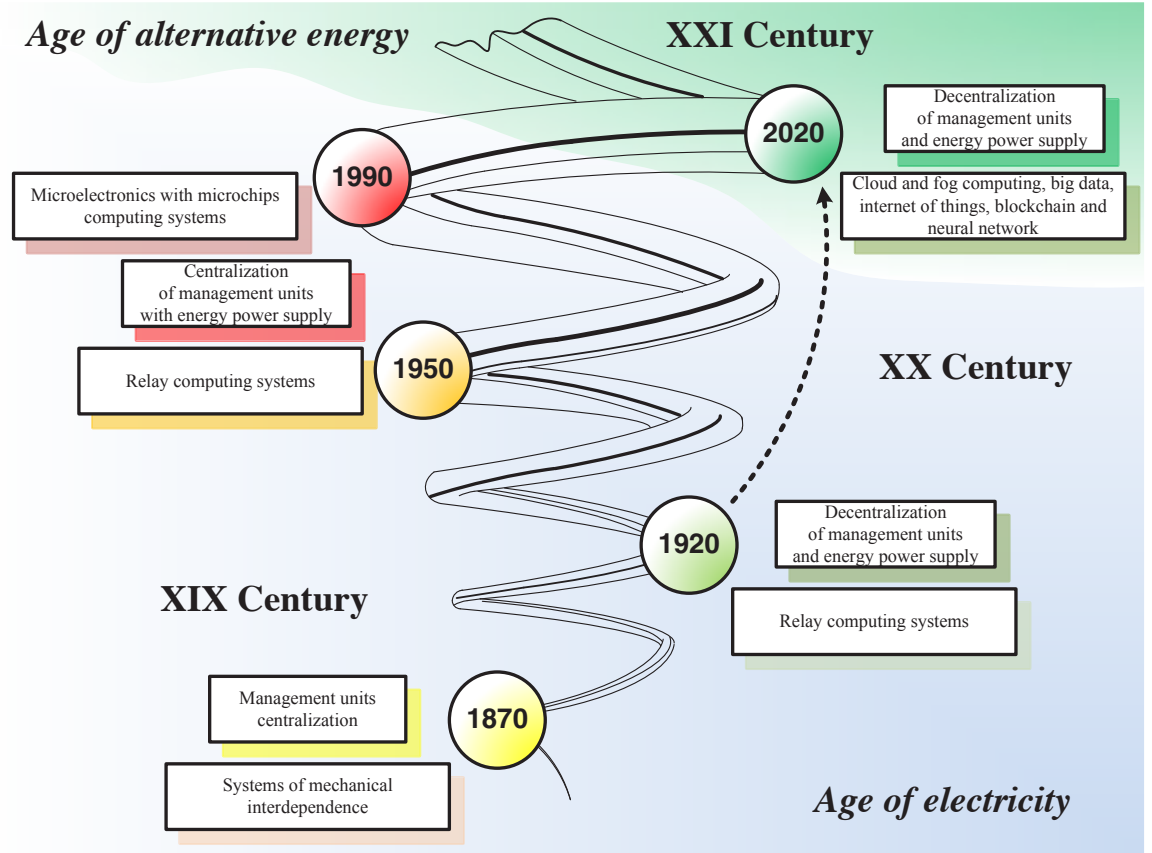

Fig. 1. Railroad management principles evolution

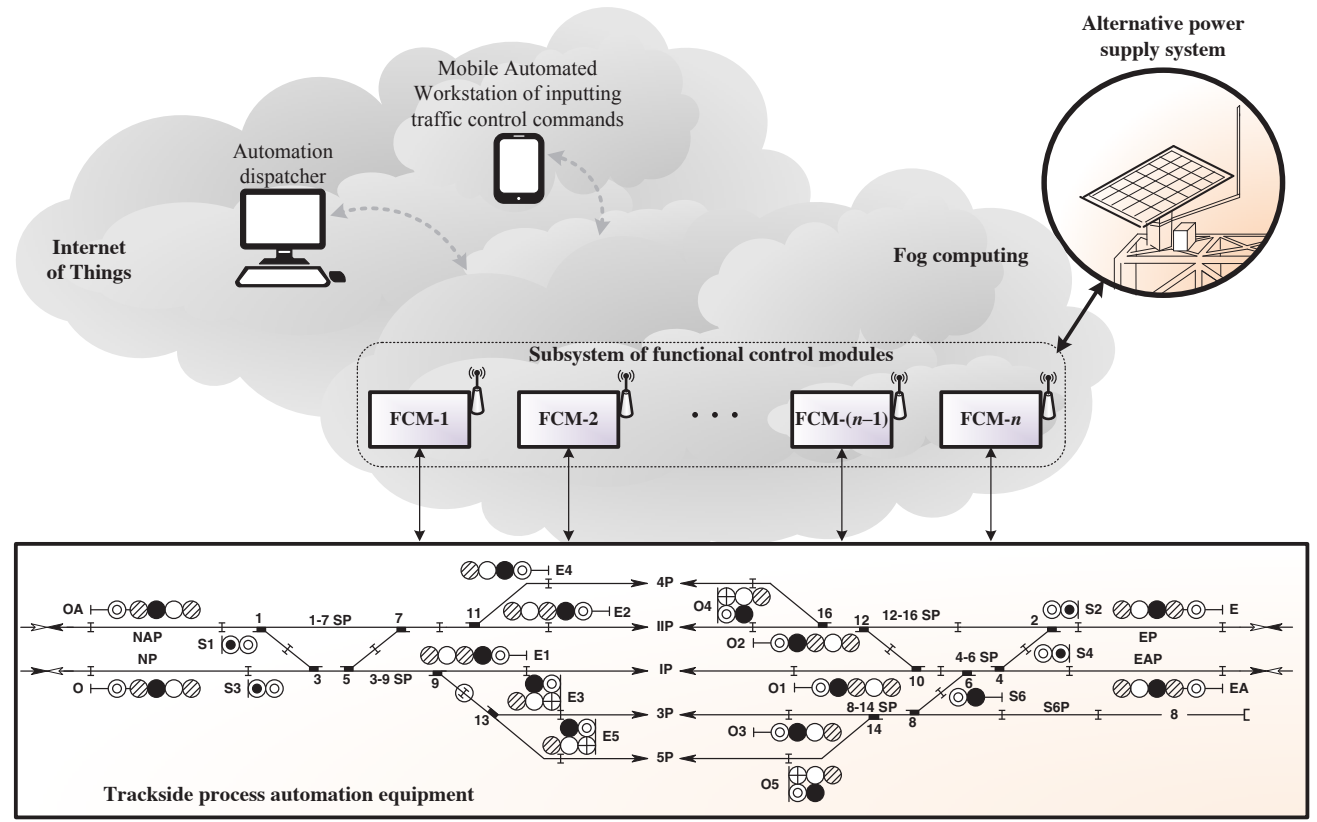

Fig 2. Green Interlocking Architecture

It should be noted that the authors of our article have already worked out the architecture similar to Green Interlocking at the railway overhead catenary monitoring sites on the St. Petersburg - Moscow High-Speed Line [16]. In the mentioned option, solar panels were installed as alternate sources of energy, and own IoT deployed on the 16kilometers' Torbino - Borovenka' railroad haul with the build-up of own secure and energyefficient data transfer protocol (Figure 3) was employed as a wireless data transmission and 
processing network. In this case, all data processing is put into practice on 'smart' sensors microcontrollers directly at the monitoring site, and the info is to be sent to the line service station concentrator on request. In the absence of a measurement procedure, the sensors are being in 'sleep mode' status quo, making a provision for energy-saving advances. The outcome of the monitoring system has shown an actual opportunity of the implemented technology performance for designing train control systems as well.

The sample of actualizing a control system having the Green Interlocking architecture at an electrified railway line station is highlighted in Figure 4. Considering the actual load on the implementation of motion control process algorithms and due regard to site geographical location, the required number of solar panels is to be analyzed in this case. Besides, IoT network with fog computing data processing technologies is being organized with the help of vertically oriented antennas and radio engineering modules of wayside automation gadgets. Total relationships are being checked at the grassroots level in a digital fog, which allows us to arrange the 'brain' of the control system into the structures that are about the size of a current smartphone (analogous to the dial-up group of electric interlocking [3]). In the long view, it shall be realistic to implement a higher level of centralization of data processing for the entire units of the railways and fulfill the transition to the utilization of artificial intelligence for train movement controlling performance.

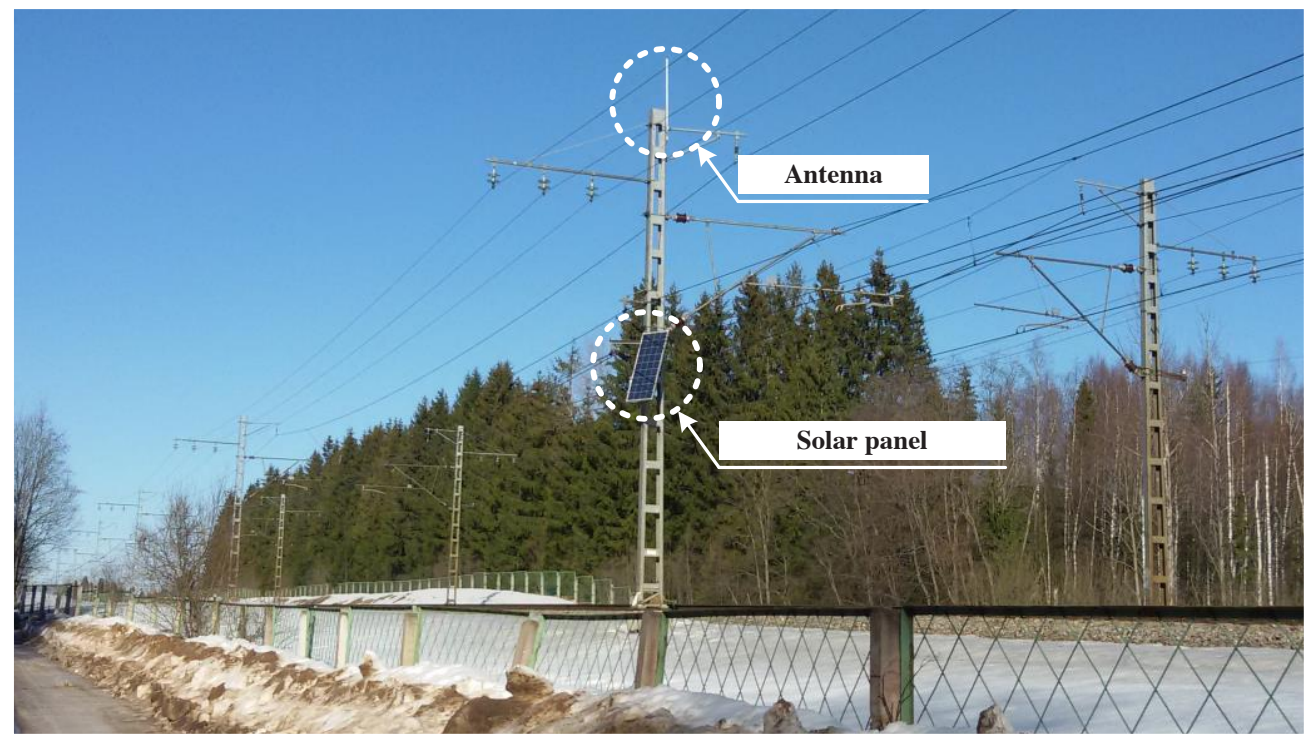

Fig. 3. Railroad haul equipment for design of Energy-Efficient Monitoring System 


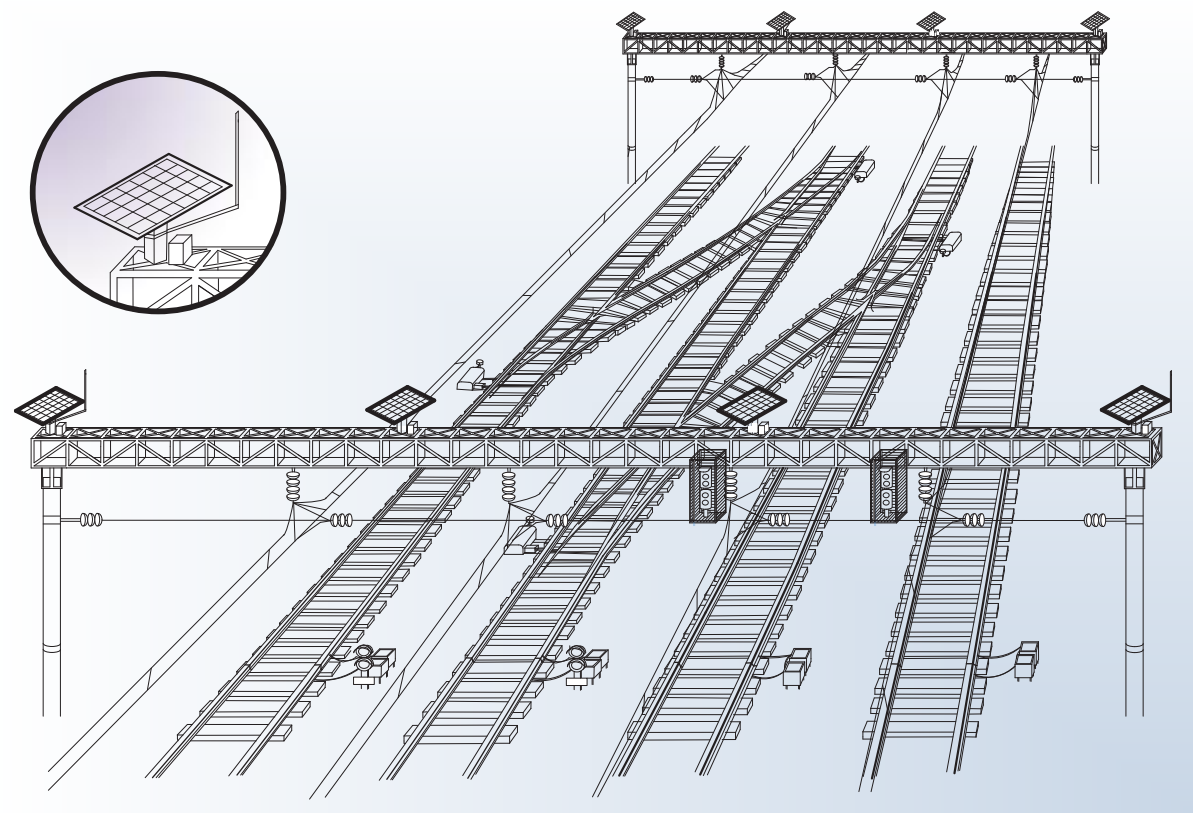

Fig. 4. Installation of solar panels with vertically directed antennas on a portal structure of a railroad overhead catenary

\section{Conclusions}

Dynamically developing information technologies allow us to unleash innovative and cutting-edge train control systems these days. The gradual transition from conventional methods and technologies of the second half of the XX Century to monitoring technologies occurs in the contemporary world. Such technologies have fully touched on space, and air transportation plus are step-by-step penetrating into the sphere of cargo and passengers ground transportation facilities, including in the railway sector.

With due regard to the prospects of alternative energy power sources and real scientific and technological progress in the field of information techs, it is a challenge to alter from classical train control kits, such as 'electric interlocking' to the accomplishment of Green Interlocking concept. This, in turn, should be a huge step frontwards the completion of Smart and Grid Train System Control. For the first time ever, the chance of forthputting and assigning energy efficiency grades toward particular railway transport control units emerge. Our experience can also be guided while designing new management systems concerning critical technological processes (or reconstruction of the old ones) being not limited to the sphere of railway transport only.

The authors of the issue have analyzed and submitted solutions per two fundamental tasks, being the main stumbling rocks of the Green Interlocking concept realization. Firstly, this is the agenda of providing security in those wireless data channels to be ensured via technologies realized by the probe of the encrypted data link for the telegram messenger (protection is so robust that the Government of the RF orders opening the transmission protocol of the designers in terms of existing strict legislation). Secondly, it is a matter of error high probability in the communication channel and the control message itself. The challenge is being solved via a new system invented by the authors by the breakthrough of fog computing tech for info processing, plus per data storage and transfer the 
abovementioned task is being solved having the idea of blockchain kit with transactions to be worked out within a set of sensors serving as a consensus mechanism.

\section{References}

1. R. Ubar, J. Raik, and H.T. Vierhaus "Design and Test Technology for Dependable Systems-on-Chip (Premier Reference Source)", Information Science Reference, Hershey - New York, IGI Global, p 578. (2011).

2. V. Kharchenko, Yu. Kondratenko, and J. Kacprzyk, Green IT Engineering: Concepts, Models, Complex Systems Architectures, Springer Book series, Studies in Systems, Decision and Control. 74, p 305, doi: 10.1007/978-3-319-44162-7. (2017)

3. G. Theeg, and S. Vlasenko "Railway Signalling \& Interlocking: 2nd edition", Germany, Hamburg: PMC Media House GmbH, p 458. (2018)

4. J. Borecky, P. Kubalik, and H. Kubatova "Reliable Railway Station System Based on Regular Structure Implemented in FPGA", 12th Euromicro Conference on Digital System Design, Architectures, Methods and Tools (DSD'09), 27-29 August 2009, Patras, Greece, pp. 348-354, doi: 10.1109/DSD.2009.210. (2009)

5. P. Novák, M. Daňhel, R.B. Blažek, M. Kohlík, and H. Kubátová "Predicting the Life Expectancy of Railway Fail-Safe Signaling Systems Using Dynamic Models with Censoring", IEEE International Conference on Software Quality, Reliability and Security (QRS), 25-29 July 2017, Prague, Czech Republic, pp. 329-339, doi: 10.1109/QRS.2017.43. (2017).

6. D. Efanov, and G. Osadchy "Paradigms for Building Control Systems on Railroad Transport: from the Systems of Electrical Interlocking of Points and Light Signals to Smart Grid Train Movements Controlling Systems", Proceedings of 16th IEEE EastWest Design \& Test Symposium (EWDTS 2018), Kazan, Russia, September 14-17, 2018, pp. 213-220.(2018)

7. V. Kharchenko, A. Gorbenko, V. Sklyar, and C. Phillips "Green Computing and Communications in Critical Application Domains: Challenges and Solutions", International Conference on Digital Technologies (DT), 29-31 May 2013, Zilina, Slovakia, pp. 191-197, doi: 10.1109/DT.2013.6566310.

8. R. Kurzweil "The Age of Intelligent Machines" Cambridge, MA: MIT Press, p. 446. ISBN 0-262-11121-7. (1990).

A. Barinova "Trains in the Netherlands Have Completely Switched to Wind Energy Usage", [http://www.nat-geo.ru/science/986915-poezda-v-niderlandakh-polnostyupereshli-na-energiyu-vetra/].

9. V.I. Hahanov, O.A. Gus, A. Ziarmand, N.C. Umerah, and A. Arefjev "Cloud Traffic Control System", Proceedings of 11th East-West Design \& Test Symposium (EWDTS 2013), Rostov-on-Don, 2013, pp. 72-76, doi: 10.1109/EWDTS.2013.6673092. (2013)

10. V. Hahanov "Cyber Physical Computing for IoT-driven Services", New York, Springer International Publishing AG, p 279, doi: 10.1007/978-3-319-54825-8. (2018).

A. Brogi, and S. Forti "QoS-Aware Deployment of IoT Applications Through the Fog", IEEE Internet of Things Journal, 4, (5), pp. 1185-1192, doi: 10.1109/JIOT.2017.2701408. (2017).

11. M.A. Serhani, H.T. El Kassabi, and I. Taleb, Quality Profile-Based Cloud Service Selection for Fulfilling Big DataProcessing Requirements", IEEE 7th International Symposium on Cloud and Service Computing (SC2), 22-25 November 2017, Kanazawa, Japan, Japan, pp. 149-156, doi: 10.1109/SC2.2017.30. (2017)

12. T.S. Darwish, and K.A. Bakar, Fog Based Intelligent Transportation Big Data Analytics in the Internet of Vehicles Environment: Motivations, Architecture, 
Challenges and Critical Issues", IEEE Access, 2018, (99), doi: 10.1109/ACCESS.2018.2815989. (2018).

13. C.H. Hsu, and U. Kremer, The Design, Implementation, and Evaluation of a Compiler Algorithm for CPU Energy Reduction, U. ACM SIGPLAN Notices, 38, (5), pp. 3848. (2003).

14. D. Efanov, G. Osadchy, D. Sedykh, D. Pristensky, and D. Barch, Monitoring System of Vibration Impacts on the Structure of Overhead Catenary of High-Speed Railway Lines", Proceedings of 14th IEEE East-West Design \& Test Symposium (EWDTS'2016), Yerevan, Armenia, October 14-17, 2016, pp. 201-208, doi: 10.1109/EWDTS.2016.7807691. (2016)

15. D. Efanov, D. Plotnikov, G. Osadchy "Prognosis Service for Navigation Systems Regarding Time Parameters of Railroad Crossing" Proceedings of 2018 IEEE EastWest Design and Test Symposium, EWDTS 2018, 8524770 doi: 10.1109/EWDTS.2018.8524770. (2018).

16. D.R. Stakhin, D.G. Plotnikov, "The Method for Determining the Risks of the Transport of Dangerous Goods" Lecture Notes in Mechanical Engineering, 2021. 194-203 doi: 10.1007/978-3-030-62062-2_19.

17. S.A. Sokolov, , D.G. Plotnikov, A.A.Grachev, V.A. Lebedev, Evaluation of loads applied on engineering structures based on structural health monitoring data, International Review of Mechanical Engineering, 2020, 14 (2), pp. 146-150. doi: 10.15866/ireme.v14i2.18269. (2020).

18. D. Efanov, D. Plotnikov, G. Osadchy, Average Number of Orders Calculation Concerning Diagnostic Test of Measuring Controllers during Permanent Monitoring Performance Based on Stationary Model of Queueing System, Proceedings of 2018 IEEE East-West Design and Test Symposium, EWDTS 2018, 8524638 doi: 10.1109/EWDTS.2018.8524638, (2018). 